\title{
Regional ionosphere map over Japanese Islands
}

\author{
J. Ping ${ }^{1}$, Y. Kono ${ }^{1}$, K. Matsumoto ${ }^{1}$, Y. Otsuka ${ }^{2}$, A. Saito ${ }^{3}$, C. Shum ${ }^{4}$, K. Heki ${ }^{1}$, and N. Kawano ${ }^{1}$ \\ ${ }^{1}$ Earth Rotation Division, National Astronomical Observatory, 023-0861, Japan \\ ${ }^{2}$ Solar-Terrestrial Environment Laboratory, Nagoya University, 464-8601, Japan \\ ${ }^{3}$ Department of Geophysics, Graduate School of Science, Kyoto University, 606-8502, Japan \\ ${ }^{4}$ Department of Civil and Environmental Engineering and Geodetic Science, The Ohio State University, 43210, U.S.A.
}

(Received September 13, 2002; Revised December 6, 2002; Accepted December 12, 2002)

\begin{abstract}
Based on the high time and spatial resolution total electron content (TEC) data, which is estimated from the phase and code observables obtained by using GPS (Global Positioning System) Earth Observation Network (GEONET), the TEC distribution and its time variation over Japanese Islands are scaled into $0.5^{\circ} \times 0.5^{\circ}$ grid data for each 10 minutes. The TEC daily map time series are arranged in an array to show the TEC evolution. Based on the spherical harmonics expansion of global ionospheric TEC model (GIM), which is estimated from global GPS observation, the TEC maps are expanded firstly through as high as 60 degrees and orders for a spherical harmonic function as a regional ionosphere map (RIM). The evolution history of medium scale traveling ionospheric disturbances (MSTIDs), i.e. polarward intense TEC enhancement and pre-noon rapid irregular fluctuations near the geomagnetic equator, are identified and confirmed in a quiet geomagnetic period.
\end{abstract}

Key words: Ionosphere, GPS, TEC, global ionosphere map, regional ionosphere map.

\section{Introduction}

Different scale ionospheric irregularities can be described by using the distributive variation of the ionospheric total electron content (TEC), which can be retrieved from the dual frequency observable of the GPS. The large scale and the medium scale ionospheric disturbances during the periods of magnetic storm have been studied in many literatures. Either global or regional GPS network has contribute to this research with different time and spatial resolution. (Wilson et al., 1995; Musman et al., 1997; Saito et al., 1998; Leonovich et al., 2000). Recently, following the increase of the density of the worldwide GPS network, and of the regional GPS networks like GEONET of Japan which has the average interstation distance as short as 25 kilometers (Miyazaki et al., 1997), the ionospheric irregularities have been studied with very high time resolution and spatial resolution as well as the high precision (Afraimovich et al., 2000; Ostuka et al., 2002).

At the global scale, ionospheric TEC distribution and variation monitored by using the worldwide GPS network is modeled by the expansion of spherical harmonic functions, also called GIM (Schaer et al., 1995). This model is released to public users in both of IONEX format and the format of spherical harmonic function coefficients at the webpage of http://www.aiub. unibe.ch/ionosphere. Since 2000, GIM has been expanded through 15 degrees and orders for every 2 hours. For GIM has been widely accepted and used in GPS and other related research fields as reference model, using the approach of GIM and taking its ad-

Copy right(c) The Society of Geomagnetism and Earth, Planetary and Space Sciences (SGEPSS); The Seismological Society of Japan; The Volcanological Society of Japan; The Geodetic Society of Japan; The Japanese Society for Planetary Sciences. vantages, in this article, a higher degrees and orders regional ionosphere map should be developed and be merged into the GIM, so as to improve the resolution of GPS TEC model in a regional area, and to use the TEC information of GIM where the regional model can not cover.

The regional GPS network of Japan, GEONET, is composed of more than 1000 GPS ground fixed receivers in Japanese Islands. GEONET is not only the powerful mission to monitor the earthquake, but also the powerful system for regional meteorological and space meteorological observe (Iwabuchi et al., 2000; Otsuka et al., 2002). A useful technique has been developed by the group of Otsuka et al. (2002) for mapping regional TEC with a spatial resolution of $0.15^{\circ} \times 0.15^{\circ}$ for every 30 seconds, from the undifferenced GPS dual frequency phase and code observable, which has been obtained by GEONET. The standard deviation of the TEC estimated from this technique is less than 1 TECU ( 3 TECU) at the northern (southern) part of Japan. 1 TECU $=1 \times 10^{16}$ electrons $/ \mathrm{m}^{2}$. The TEC obtained by using this method will be adopted in the research of this article to estimate an RIM.

\section{TEC Shell and Spherical Harmonic Function}

The ionosphere is located approximately between 70 and $1000 \mathrm{~km}$ above the surface of the earth. However, in the GPS TEC research, the ionosphere is described as a thin spherical shell model located at certain altitude with small thickness. Lots of researchers adopt a shell of altitude from $250 \mathrm{~km}$ through $450 \mathrm{~km}$, and of thickness from $200 \mathrm{~km}$ through 300 $\mathrm{km}$ based on the IRI-95 model. For the GIM model is the basic reference model in RIM estimation, the GIM TEC shell parameters of a fixed altitude of $450 \mathrm{~km}$ and a thickness of $300 \mathrm{~km}$ have been adopted in this article. 
The single-layer TEC model can either be defined in the geographical reference frame, or be defined in the geomagnetic reference frame. Because the ionospheric activity is closely related to the geomagnetic field, following the choice in GIM, the geomagnetic reference frame is adopted hereafter. The transforms between these two kinds of reference frames can easily be carried out when the geomagnetic polar is precisely defined in the geographical reference frame. The position of a point on the thin shell is described by using solar-fixed geocentric longitude and latitude pair, because the global scale ionospheric activity is almost fixed in the geomagnetic solar-fixed reference frame following the earth rotation.

In this kind of reference frame, the global or regional TEC model may be written as

$$
\begin{aligned}
\operatorname{TEC}\left(l_{a}, s\right)= & \sum_{n=0}^{n_{\max }} \sum_{m=0}^{n} P_{n m}\left(\sin l_{a}\right) \\
& *\left(a_{n m} \cos (m s)+b_{n m} \sin (m s)\right) .
\end{aligned}
$$

where $n_{\max }$ is the maximum degree of the spherical harmonic expansion; $P_{n m}\left(\sin l_{a}\right)$ are the normalized associated Legendre functions of degree $n$ and order $m ; a_{n m}$ and $b_{n m}$ are the unknown TEC coefficients of the spherical harmonics, which should be estimated; $l_{a}$ is the geocentric geomagnetic latitude of the intersection point of the receiver-satellite line with the ionospheric layer and $s$ is the solar-fixed longitude of the ionospheric pierce point, which is related to the local solar time (LT) according to

$$
s=\mathrm{LT}-12 \mathrm{~h} \approx \mathrm{UT}+l_{o}-12 \mathrm{~h} .
$$

UT is Universal Time and $l_{o}$ denotes the geographical longitude of the intersection point. Equation (1) is well suited for a regional or a global TEC model. It has been adopted to estimate the daily and sub-daily TEC model based on the global GPS surveying data set of IGS, and on the regional GPS surveying data sets obtained in North America and in Europe (Schaer et al., 1995; Wilson et al., 1995), respectively. An example of the GIM at UT01:05:00 on December 16,2001 between the geomagnetic latitude range from $0^{\circ}$ to $45^{\circ}$ is given in the top image of plate 1.

On using the GIM, slips are found between and near the boundary of two neighbor models. At ftp: / / ftp. unibe.ch/aiub/ionophere/for/, different methods of interpolation and smoothing are introduced to the user of IONEX format GIM. However, in this article, spherical harmonic function GIM model is applied into the data analysis directly. Rather than interpolating the GIM TEC at interesting point of ionospheric shell, the coefficient time series for each order and degree retrieved from the model series is interpolated into certain epoch by using a cubic spline interpolation method (Press et al., 1992). This method is effective, and a comparison of the interpolation methods will be talked in detail in another case.

\section{TEC from GEONET Observation for RIM}

The dual frequency observation by more than 1000 GPS receivers of GEONET is performed everyday. The RINEX format daily observation data is preprocessed to remove the cycle slip from the phase observable so as to obtain the relative TEC along the line of sight direction from the receiver pointing to the satellite. After that, the instrumental biases is estimated and removed from the relative TEC so as to obtain the absolute TEC for each satellite-receiver pair along the satellite track. This procedure has been described in detail by Otsuka et al. (2002), and will not be mentioned more at here. Till this step, the TEC has been obtained in geographical reference with time resolution of 30 seconds, with a potential spatial resolution of $0.15^{\circ} \times 0.15^{\circ}$. This absolute TEC is called GEOTEC hereafter. To limit the effects of ray-path bending due to the signal come from low elevation angles, only the signal with elevation angle larger than $45^{\circ}$ have been adopted.

A medium scale TEC structure from several hundreds $\mathrm{km}$ to about $1000 \mathrm{~km}$ is interested, which covers the spherical harmonic function expansion of the degrees and orders from teens through about 60 with the corresponding spatial resolution is about $3^{\circ}$. The period or lifetime of MSTID is about several tens minutes to several hours. For above research targets, the absolute TEC data has been re-scaled into $0.15^{\circ} \times 0.15^{\circ}$ grid database in geomagnetic solar-fixed reference frame for each 10 minutes. From above steps, 144 grid data files or GEOTEC maps should be obtained everyday. For each grid point in the file of given epoch, the corresponding TEC value in GIM is calculated and removed away from the map to get the residual grid data. Based on each residual grid data file, a spherical harmonic function through 60 degrees and orders could be estimated. To combine it with the spherical harmonic function model of GIM at the same epoch, a comprehensive model of RIM series with all the GIM information involved could be obtained.

\section{Some Results}

The observation data obtained by the GPS receivers of GEONET during December 2001 are processed and analyzed. The estimated GEOTEC of each 10 minutes over Japanese Islands are expanded by using a spherical harmonic function through 60 degrees and orders based on the GIM of the same sampling epoch. As an example, a combined GIM/RIM image and the corresponding GEOTEC of the same epoch of the GIM example are given just below the GIM image in Plate 1. The relatively fine structure of the RIM has been merged into GIM smoothly.

Also, a daily variation of GEOTEC has been obtained in the geomagnetic solar-fixed reference frame. The example images of 3 relative quiet geomagnetic days are read through the bottom of the Plate 1 . The daily global scale TEC variation could be identified clearly. The TEC enhancement at the middle night can be seen in the images of almost all the days in the second half month of December 2001.

The bias between GEOTEC, GIM and RIM, and the daily average of the bias are shown in Fig. 1. The large differences between GEOTEC and GIM come from two reasons. 1), when GIM is estimated, the data of only one or two GPS receivers of Japan has been used. For the larger scale structure of the ionosphere in the regional area, the poor spatial coverage of the observation data will introduce a dramatically larger bias into the GIM. See the second diagram of Fig. 1. 

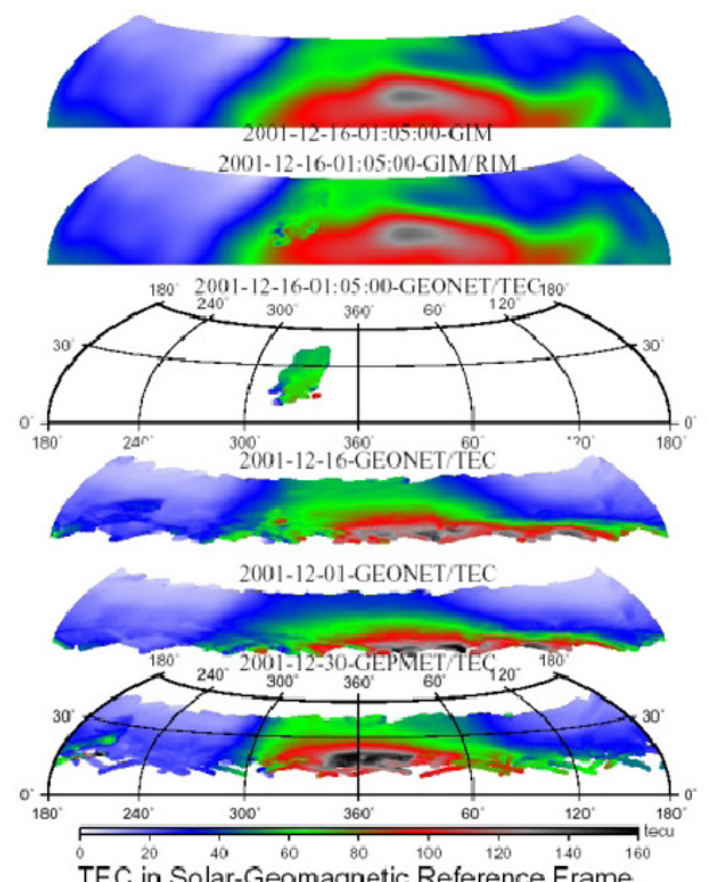

Plate 1. TEC in geomagnetic solar-fixed reference frame of latitude range from $0^{\circ}$ to $45^{\circ}$. From top through bottom: example of GIM; combined GIM/RIM; GEOTEC sampling; 3 daily GEOTEC.
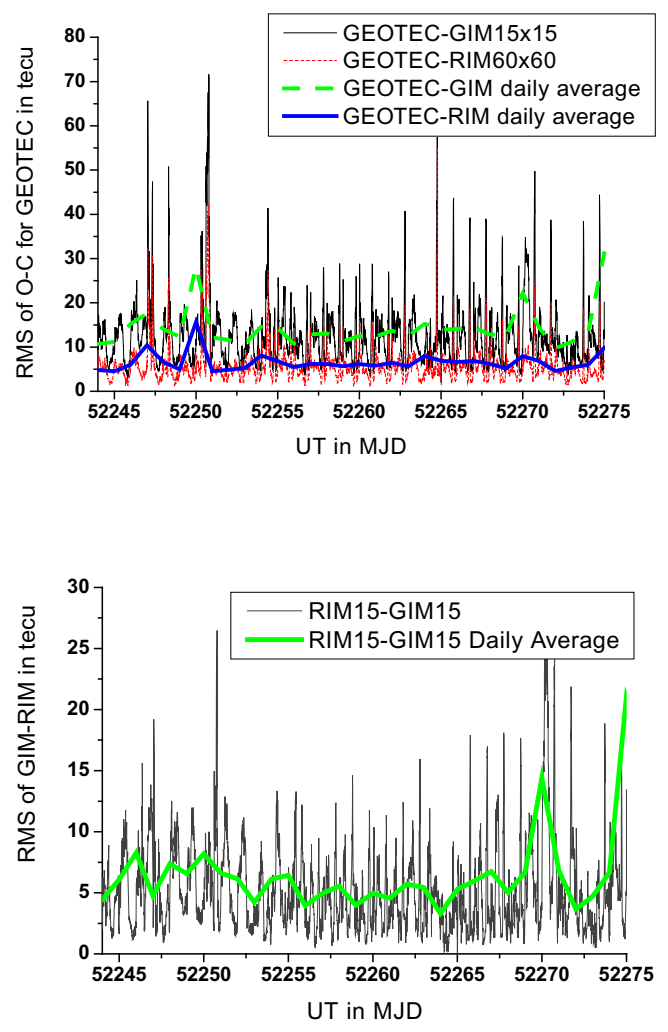

Fig. 1. Differences between GEOTEC, GIM TEC models and RIM TEC models during December 2001. Up: GEOTEC-GIM/RIM; below: RIM-GIM through 15 degrees and orders.
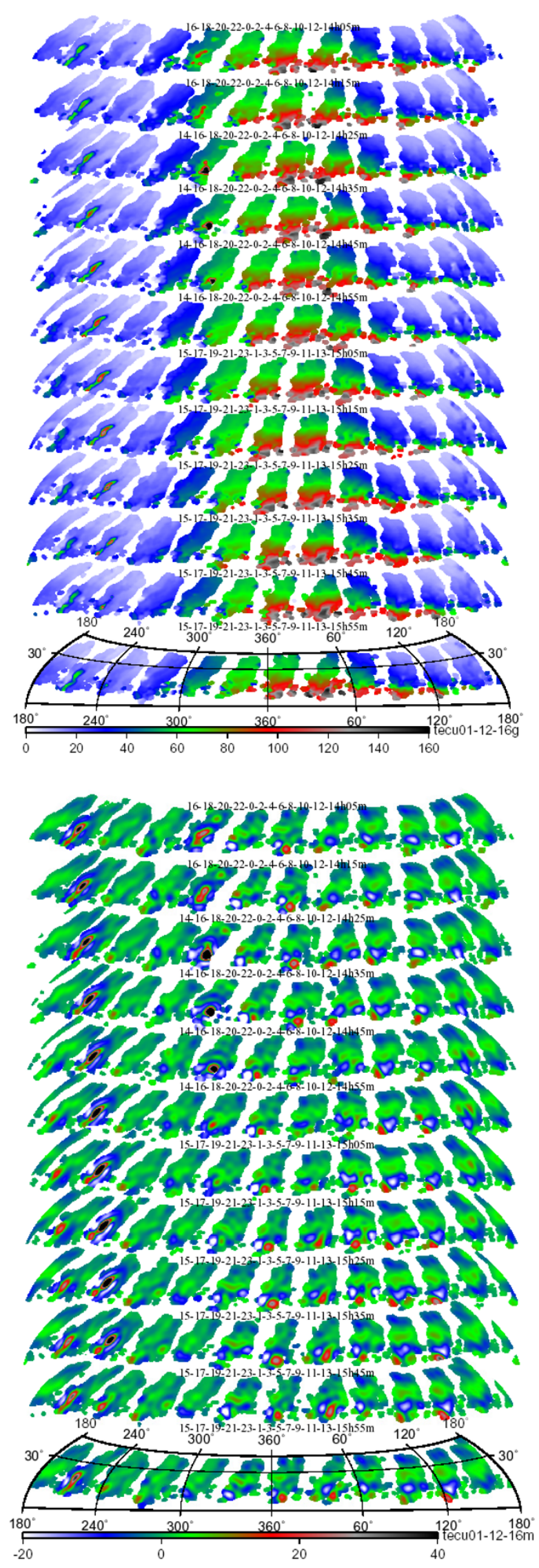

Plate 2. Snapshots array of GEOTEC of 2001/12/16 in the geomagnetic reference frame. up: TEC; below: medium scale structure. 
2), the information of TEC with period shorter than 2 hour and of scale smaller than about $1400 \mathrm{~km}$ is not included in GIM due to the large spatial and time resolution gaps. The differences in Fig. 1 also show the level of the ionospheric activities in medium and small scale. In regional area, the time and spatial resolutions of GIM can be improved by using RIM model as above.

The daily 144 high spatial resolution GEOTEC maps are arranged in the same frame of snapshots array in Plate 2. In each row of the array from right end through left end, the time increases 2 hours after each snapshot; in each 'column' of the array from top row through the bottom row, the time increases 10 minutes after each row. The corresponding epochs are given above each row. The calendar date is noted at the right bottom corner. Following the date code, 'g' replaces for GEOTEC, ' $m$ ' for medium scale structure of GEOTEC.

In the GEOTEC snapshot array, the evolution history of a middle night intense polarward TEC enhancement from geomagnetic equatorial area with period of about 3 hours and peak amplitude of 110 tecu, about 3 times denser than the background, could be identified. It has also been found in more than half of the days of December 2001. Their periods cover the range from 2 hours through 5 hours. In the array of medium scale structure, relatively weaker enhancement has been found at each night with longer periods. This phenomenon has been studied during the magnetic storm period (Otsuka et al., 2002). However, December 2001 is a quiet month of geomagnetic activity. Because this phenomenon turns around the earth with almost the same speed of the earth rotation, which means it should be connected with the regional/local geomagnetic activity other than being fixed in the geomagnetic solar-fixed reference frame. A weak magnetic storm or fluctuation is suggested to be the evolution mechanism of this phenomenon.

In the snapshot and medium scale structure image array, another kind of phenomena of pre-noon rapid TEC irregular fluctuation along the geomagnetic equatorial area, could also be identified everyday. Its period is about 4 to 5 hours from 1:00 UT with amplitude of $+/-20$ tecu. This one is connected with the solar activity, and moves parallel to equator at the low latitude area. After the TEC maximum, from afternoon through later evening, the TEC decreases other than smoothly or quietly. During this period, an afternoon TEC irregular fluctuation weaker than pre-noon fluctuation appears with longer periods from 4 through 8 hours. Its direction changes from westward to polarward, and succeeded by the night polarward enhancement at last. This kinds of medium scale periodical activities repeated everyday in December 2001 above Japanese Islands.

Using the method introduced at here, it is possible to study the small scale TEC structure and anomalies by expending the GEOTEC map into extremely high degrees and orders of spherical harmonic functions for shorter periods samplings. Long period TEC variation could also be monitored based on the long time observation. However, the narrow spatial coverage of GEONET limits the comparison with the data from other technique at broader spatial scale. It is the short come in this approach.

Acknowledgments. GEONET GPS data were provided by the Geographical Survey Institute of Japan. This work is supported by the Japan Society for the Promotion of Science, and by the SELENE/RISE project of National Astronomical Observatory. Authors also like to thank Dr. Stefan Schaer and another anonymous reviewer for their constructive comments on this work.

\section{References}

Afraimovich, E. L., E. A. Kosogorov, O. S. Lesyuta, and L. A. Leonovich, The Use of the international GPS network as the global detector (GLOBDET) simultaneously observing sudden ionospheric disturbances, Earth Planets Space, 52, 1077-1082, 2000.

Iwabushi, T., I. Naito, and N. Mannoji, A comparison of Global Positioning System retrieved precipitable water vapor with the numerical weather prediction analysis data over the Japanese Islands, J. Geophys. Res., 105, 4573-4585, 2000.

Leonovich, L. A., K. S. Palamartchouk, N. P. Perevalova, and O. M. Pirog, Observation of large-scale traveling ionospheric disturbances of auroral origin by global GPS networks, Earth Planets Space, 52, 669-674, 2000.

Miyazaki, S., T. Saito, M. Sasaki, Y. Hatanaka, and Y. Iimura, Expansion of GSI's nationwide GPS array, Bull. Geogr. Surv. Inst., 43, 23-34, 1997.

Musman, S., J.-M. Jahn, J. LaBelle, and W. E. Swartz, Imaging spreadF structures using GPS observations at Alcantara, Brazil, Geophy. Res. Lett., 24, 1703-1706, 1997.

Otsuka, Y., T. Ogawa, A. Saito, T. Tsugawa, S. Fukao, and S. Miyazaki, A new technique for mapping of total electron content using GPS network in Japan, Earth Planets Space, 54, 63-70, 2002.

Press, W. H., S. A. Teukolsky, W. T. Vetterling, and B. P. Flannery, Cubic Spline Interpolation in Numerical Recipes in Fortran, Second Edition, 107 pp., Cambridge University Press, Cambridge, 1992.

Saito, A., S. Fukao, and S. Miyazaki, High resolution mapping of TEC perturbations with the GSI GPS network over Japan, Geophys. Res. Lett., 25, 3079-3082, 1998.

Schaer, S., G. Beutler, L. Mervart, M. Rothacher, and U. Wild, Global and Regional Ionosphere Models Using the GPS Double Difference Phase Observable, Proceedings of the IGS Workshop on Special Topics on New Directions, 77-92 Potsdam, Germany, May 15-17, 1995.

Wilson, B. D., A. J. Mannucci, and C. D. Edwards, Subdaily northern hemisphere ionospheric maps using an extensive network of GPS receivers, Radio Sci., 30, 639-648, 1995.

J. Ping (e-mail: jsping@miz.nao.ac.jp), Y. Kono, K. Matsumoto, Y. Otsuka, A. Saito, C. Shum, K. Heki, and N. Kawano 